\title{
$\mathrm{MgO} / \mathrm{SrO} / \mathrm{La}_{2} \mathrm{O}_{3}$ 多元添加对 $\left(\mathrm{Zr}_{0.8} \mathrm{Sn}_{0.2}\right) \mathrm{TiO}_{4}$ 微波陶瓷结构性能影响
}

\author{
孙庆䂞 ${ }^{1}$, 周洪庆 ${ }^{1}$, 钱 䂞 ${ }^{1}$, 王亚洲 ${ }^{1}$, 朱海奎 ${ }^{1}$, 岳振星 ${ }^{2}$
} (1. 南京工业大学 材料科学与工程学院, 南京 $210009 ; 2$. 清华大学 新型陶瓷与精细工艺国家重点实验室, 北京 100084)

摘 要: 基于液相促进固相反应烧结机制, 设计 $\mathrm{MgO} / \mathrm{SrO} / \mathrm{La}_{2} \mathrm{O}_{3}$ 多元复合添加 $\left(\mathrm{Zr}_{0.8} \mathrm{Sn}_{0.2}\right) \mathrm{TiO}_{4}(\mathrm{ZST})$ 体系, 探究复合 添加剂对 ZST 陶瓷的物相组成、微观结构、烧结特性以及高频介电性能等参数的影响。实验结果表明: 陶瓷的主 晶相均为 ZST 相; 适量添加 $\mathrm{MgO} / \mathrm{SrO} / \mathrm{La}_{2} \mathrm{O}_{3}$ 可以有效地降低 ZST 陶瓷的烧结温度, 获得较优的微波介电性能; 但 $\mathrm{MgO}$ 添加量的增多对材料的综合性能有小幅度的影响; $\mathrm{SrO}$ 的添加量过大会造成晶粒的不完全生长、瓷体不致密和 气孔的增多, 从而导致材料的密度、介电常数和 $Q \times f$ 值的下降; 此外, 添加剂对陶瓷的频率温度系数 $\left(\tau_{\mathrm{f}}\right)$ 影响不大。 在复合添加 $0.2 \mathrm{wt} \% \mathrm{MgO} 、 0.6 \mathrm{wt} \% \mathrm{SrO} 、 1.0 \mathrm{wt} \% \mathrm{La}_{2} \mathrm{O}_{3}$ 时, $1300{ }^{\circ} \mathrm{C}$ 保温 $5 \mathrm{~h}$ 的 ZST 陶瓷综合性能优异: $\rho=5.14 \mathrm{~g} / \mathrm{cm}^{3}$, $\varepsilon_{\mathrm{r}}=40.11, Q \times f=51000 \mathrm{GHz}(f=5.61 \mathrm{GHz}), \tau_{\mathrm{f}}=-2.85 \times 10^{-6{ }^{\circ} \mathrm{C}^{-1}}$ 。

关 键 词: $\left(\mathrm{Zr}_{0.8} \mathrm{Sn}_{0.2}\right) \mathrm{TiO}_{4} ; \mathrm{MgO} / \mathrm{SrO}$ 多元添加; 微观结构; 烧结特性; 介电性能

中图分类号: TB34 文献标识码: A

\section{Effects of $\mathrm{MgO}$, $\mathrm{SrO}$ and $\mathrm{La}_{2} \mathrm{O}_{3} \mathrm{Co}$-doping on Structure and Properties of $\left(\mathrm{Zr}_{0.8} \mathrm{Sn}_{0.2}\right) \mathrm{TiO}_{4}$ Ceramics}

\author{
SUN Qing-Lei ${ }^{1}$, ZHOU Hong-Qing ${ }^{1}$, QIAN-Lei ${ }^{1}$, WANG Ya-Zhou ${ }^{1}$, ZHU Hai-Kui ${ }^{1}$, YUE Zhen-Xing ${ }^{2}$ \\ (1. School of Materials Science and Engineering, Nanjing Tech University, Nanjing 210009 China; 2. State Key Laboratory of \\ New Ceramics and Fine Processing, Tsinghua University, Beijing 100084, China)
}

\begin{abstract}
Zr}_{0.8} \mathrm{Sn}_{0.2}\right) \mathrm{TiO}_{4}(\mathrm{ZST})$ ceramics were fabricated via conventional solid-state reaction method which was promoted by liquid phase. The effects of $\mathrm{MgO}, \mathrm{SrO}$ and $\mathrm{La}_{2} \mathrm{O}_{3}$ co-doping ZST ceramics on phase composition, microstructures, sintering behavior, and high frequency dielectric properties were investigated systematically. The results showed that the main crystalline phase of the ceramics was ZST. The appropriate amount of $\mathrm{MgO}, \mathrm{SrO}$ and $\mathrm{La}_{2} \mathrm{O}_{3}$ additions effectively decreased the sintering temperature of ZST ceramics and improved the microwave dielectric properties. The increment of $\mathrm{MgO}$ addition influenced comprehensive properties of ZST ceramics slightly. The decrement in bulk densities, dielectric constants and $Q \times f$ values with excessive $\mathrm{SrO}$ addition was due to incomplete growth of grains, incompact structures and increasing porosities. Additionally, the additives did not affect obviously the temperature coefficient of resonant frequency $\left(\tau_{\mathrm{f}}\right)$ of the ceramics. When added $0.2 \mathrm{wt} \% \mathrm{MgO}, 0.6 \mathrm{wt} \%$ $\mathrm{SrO}$ and $1.0 \mathrm{wt} \% \mathrm{La}_{2} \mathrm{O}_{3}$ and sintered at $1300^{\circ} \mathrm{C}$ for $5 \mathrm{~h}, \mathrm{ZST}$ ceramics displayed the optimum comprehensive prop-
\end{abstract}

收稿日期: 2015-12-15; 收到修改稿日期：2016-02-14

基金项目: 江苏省 2015 年度普通高校研究生实践创新计划项目(SJZZ15_0095); 教育部创新团队项目(IRT_15R35); 江苏高 校优势学科建设工程资助项目(PAPD)

Practice Innovation Program (2015) for University Graduate Students of Jiangsu Province (SJZZ15_0095); Program for Innovative Research Team in University of Ministry of Education of China (IRT_15R35); Priority Academic Program Development of Jiangsu Higher Education Institution (PAPD)

作者简介: 孙庆磊(1991-), 男, 硕士研究生. E-mail: lijia20162017@163.com

通讯作者：周洪庆，教授.E-mail: hqzhou@njtech.edu.cn 
erties: $\rho=5.14 \mathrm{~g} / \mathrm{cm}^{3}, \varepsilon_{\mathrm{r}}=40.11, Q \times f=51000 \mathrm{GHz}(f=5.61 \mathrm{GHz}), \tau_{\mathrm{f}}=-2.85 \times 10^{-6{ }^{\circ} \mathrm{C}^{-1}}$.

Key words: $\left(\mathrm{Zr}_{0.8} \mathrm{Sn}_{0.2}\right) \mathrm{TiO}_{4}$; $\mathrm{MgO}$, SrO co-doping; microstructures; sintering behavior; dielectric properties

近年来以移动通信、北斗卫星导航、直播卫星 DBS、 汽车电子等为代表的高频微波技术发展迅速 ${ }^{[1,2]}$, 高端信息技术向多功能集成化、大功率小型化等方 向发展已成为主流 ${ }^{[3]}$ 。随着高频无源有源电路集成 化程度的提高, 对宽频微波介质材料的 $Q$ 值提出了 更高的要求。 $\left(\mathrm{Zr}_{0.8} \mathrm{Sn}_{0.2}\right) \mathrm{TiO}_{4}(\mathrm{ZST})$ 陶瓷以其优异的 微波介电性能, 一直是人们研究的热点之一 ${ }^{[4-7]}$ 。

NEDELCU 等 ${ }^{[8]}$ 在 ZST 陶瓷中添加 $0.2 \mathrm{wt} \%$ 的 $\mathrm{MgO}$, 在 $1315^{\circ} \mathrm{C}$ 烧结 $4 \mathrm{~h}$ 得到 $\varepsilon_{\mathrm{r}}=36.6, Q \times f$ 值达 61000 的陶瓷。IOACHIM 等 ${ }^{[9]}$ 在 ZST 陶瓷中添加 $0.2 \mathrm{wt} \%$ $\mathrm{MgO} 、 1 \mathrm{wt} \% \mathrm{ZnO}$ 和 $2 \mathrm{wt} \% \mathrm{La}_{2} \mathrm{O}_{3}$, 在 $1320^{\circ} \mathrm{C}$ 保温 $2 \mathrm{~h}$, 制备出 $\varepsilon_{\mathrm{r}}=36.7, Q=9200$ 的高 $Q$ 陶瓷。ZHANG 等 ${ }^{[10]}$ 添加 $1 \mathrm{wt} \% \mathrm{La}_{2} \mathrm{O}_{3}$ 和 $2 \mathrm{wt} \% \mathrm{BaO}$ 改性 ZST 陶瓷, $1350^{\circ} \mathrm{C}$ 保温 $2 \mathrm{~h}$, 获得了体密度 $\rho=5.10 \mathrm{~g} / \mathrm{cm}^{3}, \varepsilon_{\mathrm{r}}=41, Q=$ 9800 的高性能陶瓷。

在前期研究的基础上, 本工作进一步引入 $\mathrm{SrO}$, 通过对 ZST 陶瓷体系的多元复合添加, 以期系统地 研究烧结特性好、综合性能更好的微波中介陶瓷。

\section{1 实验方法}

\section{1 样品制备}

精选分析纯原料, 固相反应合成 ZST 粉, 其中 $\mathrm{ZrO}_{2}$ (纯度 $>99 \%$ )、 $\mathrm{SnO}_{2}$ (纯度 $>99 \%$ )、 $\mathrm{TiO}_{2}$ (纯度> $99 \%$ )、 $\mathrm{La}_{2} \mathrm{O}_{3}$ (纯度 $>99 \%$ )、 $\mathrm{MgO}$ (纯度 $>99 \%$ ) 和 $\mathrm{SrCO}_{3}$ (纯度 $>99 \%$ )。制备陶瓷的化学通式设计如下: $\left(\mathrm{Zr}_{0.8} \mathrm{Sn}_{0.2}\right) \mathrm{TiO}_{4}+x \mathrm{wt} \% \mathrm{MgO}+y \mathrm{wt} \% \mathrm{SrO}+1 \mathrm{wt} \% \mathrm{La}_{2} \mathrm{O}_{3}$, (式中 $x=0.2 、 0.4 ; y=0.2 、 0.4 、 0.6 、 0.8 、 1.0$ )。

首先按照 ZST 化学计量比配比、称量、混合 $24 \mathrm{~h}$, 在 $1050^{\circ} \mathrm{C}$ 预烧 $3 \mathrm{~h}$, 破碎粉碎预烧块; 将一定量的 $\mathrm{MgO} 、 \mathrm{SrO} 、 \mathrm{La}_{2} \mathrm{O}_{3}$ 加入到细磨后的预烧料中, 其中 $\mathrm{SrO}$ 通过 $\mathrm{SrCO}_{3}$ 在 $1100^{\circ} \mathrm{C}$ 下分解所得, 配料以氧化 锆球和去离子水作为研磨介质一起装入尼龙罐中, 用行星磨球机球磨 $12 \mathrm{~h}$, 烘干后的粉料加入 $8 \mathrm{wt} \%$ 浓度为 7\%的 PVA 水溶液造粒, 在 $100 \mathrm{MPa}$ 压力下 将粉料压制成 $\phi 13.1 \mathrm{~mm} \times(6.5 \sim 7.5) \mathrm{mm}$ 的圆片, 并在 $1280^{\circ} \mathrm{C} 、 1300^{\circ} \mathrm{C} 、 1320^{\circ} \mathrm{C} 、 1340^{\circ} \mathrm{C}$ 进行烧结, 保温 时间为 $5 \mathrm{~h}$, 然后随炉冷却至室温。各组 $\mathrm{MgO} / \mathrm{SrO}$ 质量分数及编号如表 1 所示。

\section{2 性能检测}

采用阿基米德排水法测试试样的体积密度。用 日本理学公司 $\mathrm{SmartLab}$ 型X线衍射仪对试样进行
表 1 不同 $\mathrm{MgO} / \mathrm{SrO}$ 添加量试样及其编号 Table 1 Samples with different mass fraction of $\mathrm{MgO} / \mathrm{SrO}$

\begin{tabular}{ccc}
\hline Sample & $\mathrm{MgO} / \mathrm{wt} \%$ & $\mathrm{SrO} / \mathrm{wt} \%$ \\
\hline $\mathrm{A}_{1}$ & 0.2 & 0.2 \\
$\mathrm{~A}_{2}$ & 0.2 & 0.4 \\
$\mathrm{~A}_{3}$ & 0.2 & 0.6 \\
$\mathrm{~A}_{4}$ & 0.2 & 0.8 \\
$\mathrm{~A}_{5}$ & 0.2 & 1.0 \\
$\mathrm{~B}_{1}$ & 0.4 & 0.2 \\
$\mathrm{~B}_{2}$ & 0.4 & 0.4 \\
$\mathrm{~B}_{3}$ & 0.4 & 0.6 \\
$\mathrm{~B}_{4}$ & 0.4 & 0.8 \\
$\mathrm{~B}_{5}$ & 0.4 & 1.0 \\
\hline
\end{tabular}

$\mathrm{XRD}$ 分析，扫描速度为 $10 \% \mathrm{~min}$ 。用 JSM-5900 型扫 描电镜观察试样的微观形貌。用美国 Agilent 公司 $8722 \mathrm{ET}$ 型网络分析仪测定试样高频性能; 采用 RGWT-4002 型万能试验机, 用三点弯曲法和单边 切口梁法测量样品的抗弯强度, 抗弯强度的加载速 度为 $0.5 \mathrm{~mm} / \mathrm{min}$ 。用美国 Agilent 公司 $8722 \mathrm{ET}$ 型网 络分析仪与精密恒温箱, 在 $25 \sim 80^{\circ} \mathrm{C}$ 温度范围内测 量试样的谐振频率温度系数 $\tau_{\mathrm{f}}$, 其计算公式为:

$$
\tau_{\mathrm{f}}=\frac{1}{f_{25}} \times \frac{f_{80}-f_{25}}{80-25}
$$

其中: $f_{25}$ 与 $f_{80}$ 分别为 $25^{\circ} \mathrm{C}$ 和 $80^{\circ} \mathrm{C}$ 下的谐振频率。

\section{2 结果与讨论}

\section{1 物相分析}

图 1(A)为不同 $\mathrm{MgO} / \mathrm{SrO}$ 添加量 $\mathrm{ZST}$ 陶瓷的 $\mathrm{XRD}$ 图谱。添加少量 $\mathrm{MgO} / \mathrm{SrO}$, 样品中都生成了 单一的 ZST 晶相, 属斜方晶系。XRD 没有检测出 第二相。由图 1(B)可以看出, 当 $\mathrm{SrO}$ 的添加量由 $0.2 \mathrm{wt} \%$ 增加到 $0.6 \mathrm{wt} \%$, 在烧结温度为 $1300^{\circ} \mathrm{C}$ 时， 样品的(111)晶面衍射峰偏移幅度较小; 当 $\mathrm{SrO}$ 的 添加量为 $1.0 \mathrm{wt} \%$, 在烧结温度为 $1280^{\circ} \mathrm{C}$ 时, , 样品 的(111)晶面衍射峰向较高角度偏移, 表 2 列出根据 XRD 数据所计算得出的晶格常数和晶胞体积。在 $1300^{\circ} \mathrm{C}$ 烧结的 $\mathrm{A}_{1} 、 \mathrm{~A}_{3}$ 试样晶格常数 $a 、 b 、 c$ 以及 晶胞体积基本没有变化, 而 $1280^{\circ} \mathrm{C}$ 烧结的 $\mathrm{A}_{5}$ 试样 晶格常数 $a$ 显著增大, $b 、 c$ 略微减小, 同时晶胞体 积明显增大。 

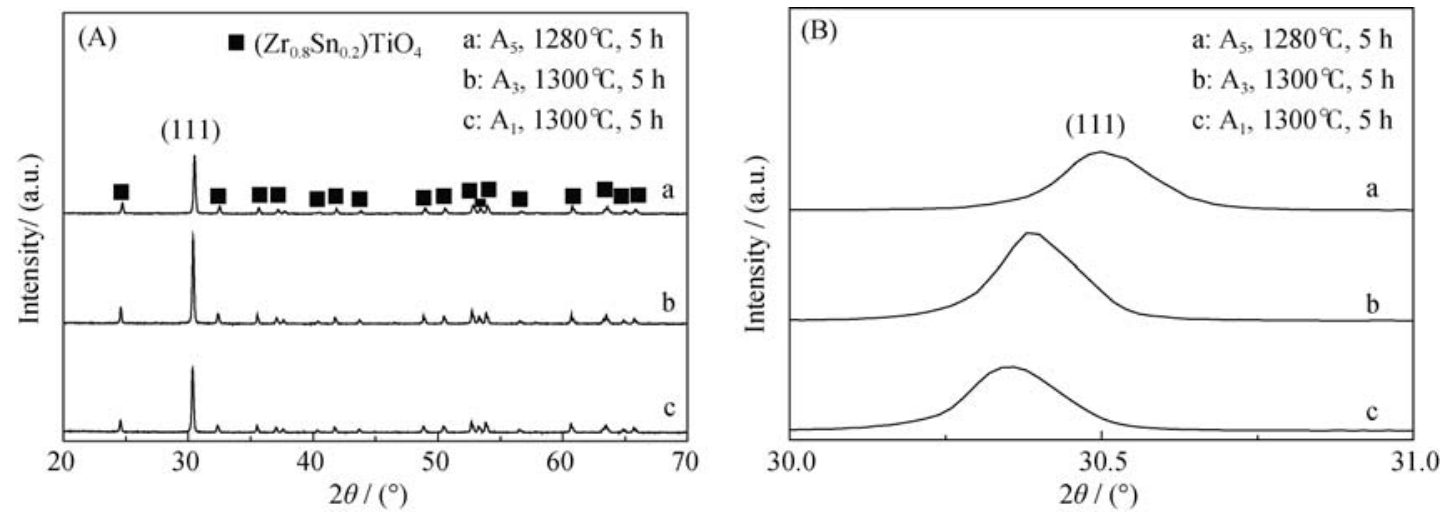

图 1 ZST 试样的 XRD 图谱

Fig. 1 XRD patterns of ZST specimens

表 2 ZST 试样的晶格常数和晶胞体积

Table 2 Lattice parameters and unit cell volume of ZST specimens

\begin{tabular}{cccccc}
\hline Sample & $\begin{array}{c}\text { Sintering tem- } \\
\text { perature } /{ }^{\circ} \mathrm{C}\end{array}$ & $a / \mathrm{nm}$ & $b / \mathrm{nm}$ & $c / \mathrm{nm}$ & $\begin{array}{c}\text { Unit cell } \\
\text { volume } / \mathrm{nm}^{3}\end{array}$ \\
\hline $\mathrm{A}_{1}$ & 1300 & 0.4471 & 0.5050 & 0.558 & 0.12599 \\
$\mathrm{~A}_{3}$ & 1300 & 0.4473 & 0.5050 & 0.558 & 0.12604 \\
$\mathrm{~A}_{5}$ & 1280 & 0.4806 & 0.5032 & 0.5447 & 0.13173 \\
\hline
\end{tabular}

\section{2 微观形貌}

图 2 为不同温度烧结 ZST 试样的断面 SEM 照 片。 $1280^{\circ} \mathrm{C}$ 烧结的 $\mathrm{A}_{1}$ 试样晶粒发育良好, 晶界较为 清晰, 平均粒径 $3 \sim 5 \mu \mathrm{m}$; 而 $1300^{\circ} \mathrm{C}$ 烧结的 $\mathrm{A}_{3}$ 试样, 相比 $\mathrm{A}_{1}$ 试样, 具有明显的柱状晶粒, 晶粒发育更加 饱满, 晶粒尺寸有所增大, 平均为 $5 \sim 8 \mu \mathrm{m}$, 结构均
匀、致密; 当烧结温度升高到 $1340^{\circ} \mathrm{C}$ 时, 由于烧结 温度过高使 $\mathrm{A}_{3}$ 试样的某些晶粒产生了异常生长的 现象, 较大的颗粒粒径可达 $8 \sim 10 \mu \mathrm{m}$, 而较小的仅 为 2 3 $\mu \mathrm{m}$, 并且产生了少量的气孔, 气孔直径约为 0.5 1 $\mu \mathrm{m}$, 晶界变得稍有模糊, 结构均匀性变差, 致密程度有所降低。1280 ${ }^{\circ} \mathrm{C}$ 烧结的 $\mathrm{A}_{5}$ 试样的晶粒致 密性较差, 晶界与晶界之间产生了气孔，由于大量 液相存在，导致晶粒模糊; 而当烧结温度达到 $1340^{\circ} \mathrm{C}$ 时, $\mathrm{A}_{5}$ 试样结构变得较为致密, 但还是难以 看出较为明显的晶粒, 并且同样有较多的气孔与液 相存在。当 $\mathrm{MgO}$ 添加量由 $0.2 \mathrm{wt} \%$ 增加到 $0.4 \mathrm{wt} \%$, 对 比图 2(e、f)可以看出, 在 $1340^{\circ} \mathrm{C}$ 烧结的 $\mathrm{B}_{5}$ 试样有 较多的气孔，小部分晶粒已经扭曲变形，而相同
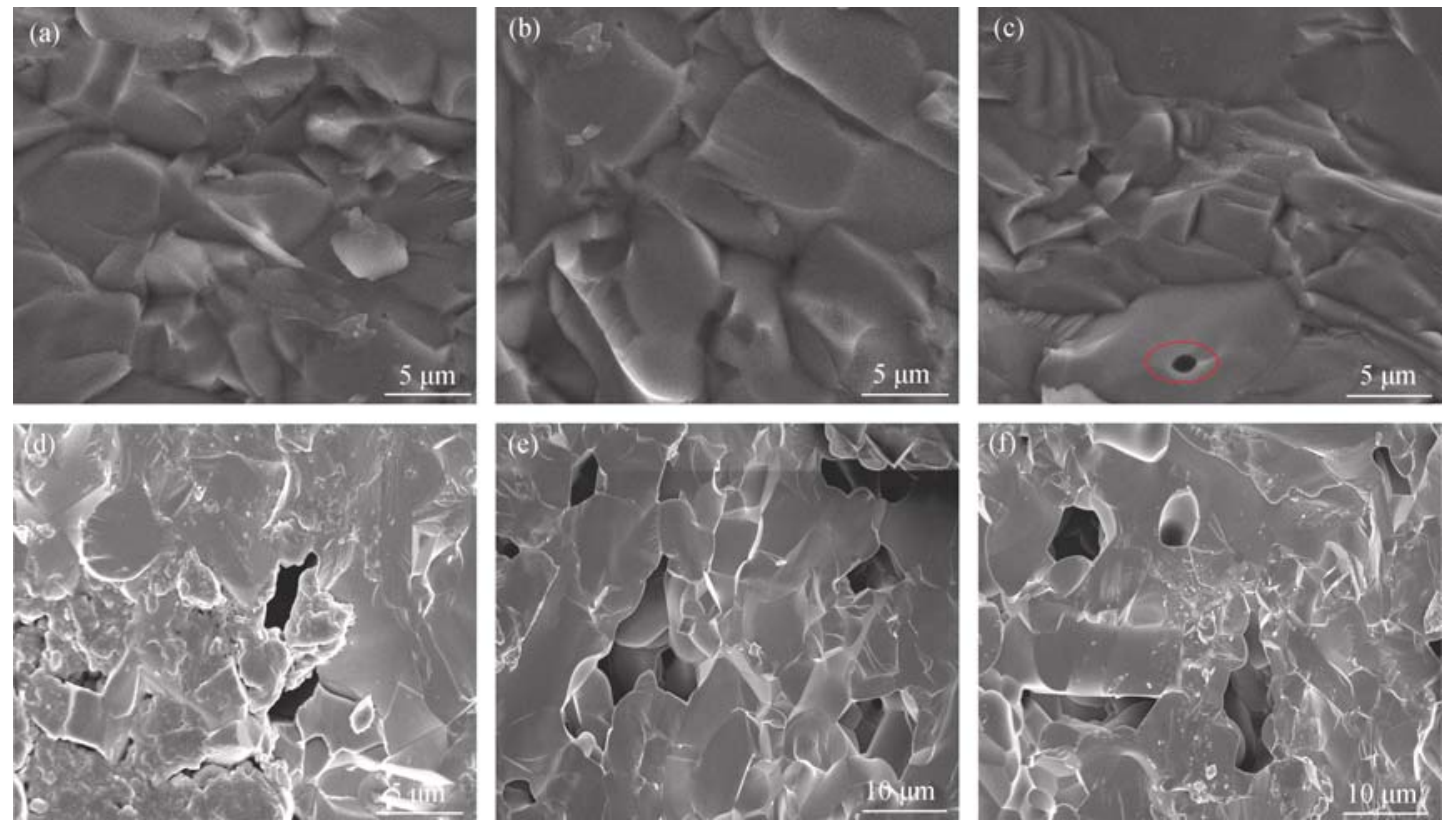

图 2 不同温度烧结 ZST 试样的断面 SEM 照片

Fig. 2 Fracture surface SEM images of ZST specimens sintered at different temperatures (a) $\mathrm{A}_{1}$ sintered at $1280^{\circ} \mathrm{C}$; (b) $\mathrm{A}_{3}$ sintered at $1300^{\circ} \mathrm{C}$; (c) $\mathrm{A}_{3}$ sintered at $1340^{\circ} \mathrm{C}$; (d) $\mathrm{A}_{5}$ sintered at $1280^{\circ} \mathrm{C}$; (e) $\mathrm{A}_{5}$ sintered at $1340^{\circ} \mathrm{C}$; (f) $\mathrm{B}_{5}$ sintered at $1340^{\circ} \mathrm{C}$ 
温度烧结的 $\mathrm{A}_{5}$ 试样具有更好的致密性和晶粒形貌, 并且液相较少。

\section{3 烧结特性}

图 3(a、b)为 $\mathrm{MgO}$ 的添加量为 $0.2 \mathrm{wt} \% 、 0.4 \mathrm{wt} \%$, 对应 $\mathrm{SrO}$ 添加量分别为 $0.2 \mathrm{wt} \% 、 0.4 \mathrm{wt} \% 、 0.6 \mathrm{wt} \%$ 、 $0.8 \mathrm{wt} \% 、 1.0 \mathrm{wt} \%$, 烧结温度范围为 $1280 \sim 1340^{\circ} \mathrm{C}$, 所 得试样密度随着烧结温度的变化。当 $\mathrm{SrO}$ 的添加量 为 $0.2 \mathrm{wt} \% 、 0.4 \mathrm{wt} \% 、 0.6 \mathrm{wt} \%$, 在 $1300^{\circ} \mathrm{C}$ 烧结时, $\mathrm{A}_{1}$ 、 $\mathrm{A}_{2} 、 \mathrm{~A}_{3}$ 试样的密度变化不大, 基本维持在 $5.1 \mathrm{~g} / \mathrm{cm}^{3}$ 左右。 $\mathrm{A}_{3}$ 试样的最大密度为 $5.14 \mathrm{~g} / \mathrm{cm}^{3}$, 达到理论 密度 $\left(5.19 \mathrm{~g} / \mathrm{cm}^{3}\right)$ 的 $99.04 \% ; B_{3}$ 试样的最大密度为 $5.12 \mathrm{~g} / \mathrm{cm}^{3}$, 达到理论密度的 $98.65 \%$ 。当烧结温度由 $1280^{\circ} \mathrm{C}$ 升高到 $1340^{\circ} \mathrm{C}$, 试样的密度呈现先增大后减 小的变化趋势。由图 2(a、b、c)可以看出, 在 1300 ${ }^{\circ} \mathrm{C}$ 烧结的 $\mathrm{A}_{3}$ 试样, 形貌比较完善; 在 $1280^{\circ} \mathrm{C}$ 烧结 $\mathrm{A}_{1}$ 试样和在 $1300^{\circ} \mathrm{C}$ 烧结 $\mathrm{A}_{3}$ 试样的形貌比较相近; 而在 $1340^{\circ} \mathrm{C}$ 烧结 $\mathrm{A}_{3}$ 试样形貌明显变差。当 $\mathrm{SrO}$ 的 添加量为 $0.8 \mathrm{wt} \% 、 1.0 \mathrm{wt} \%$, 在 $1280^{\circ} \mathrm{C}$ 烧结时, $\mathrm{A}_{5}$ 试样的密度仅为 $4.24 \mathrm{~g} / \mathrm{cm}^{3}, B_{5}$ 试样的密度仅为 $4.22 \mathrm{~g} / \mathrm{cm}^{3}$; 在 $1340^{\circ} \mathrm{C}$ 烧结时, $\mathrm{A}_{5} 、 \mathrm{~B}_{5}$ 试样的密度分 别可以达到 4.82 和 $4.85 \mathrm{~g} / \mathrm{cm}^{3}$ 。在 $1280^{\circ} \mathrm{C}$ 烧结时, 试 样的密度较小, 随着烧结温度的升高, 试样的密度 逐渐增大。对比图 2(d、e) 可以看出, 当烧结温度升 高至 $1340^{\circ} \mathrm{C}$ 时, $\mathrm{A}_{5}$ 试样烧结更致密, 晶粒发育相对 更好。WANG 等 ${ }^{[11]}$ 曾指出: 由于 $\mathrm{Sr}^{2+}(0.118 \mathrm{~nm})$ 的半 径远远大于 $\mathrm{Zr}^{4+}(0.072 \mathrm{~nm}) 、 \mathrm{Sn}^{4+}(0.069 \mathrm{~nm}) 、 \mathrm{Ti}^{4+}$ $(0.061 \mathrm{~nm})$, 在 ZST 陶瓷烧结过程中, $\mathrm{Sr}^{2+}$ 进入晶格 后不会取代这三种离子, 也不可能置换其他阳离子, 所以只能进入晶格的间隙位置。这将会使 ZST 试样 的晶格发生比较大的形变, 内部空间结构也因此变 得比较松弛, 从而使 $\mathrm{Zr}^{4+} 、 \mathrm{Sn}^{4+} 、 \mathrm{Ti}^{4+}$ 三种离子的空 间势阱降低, 离子运动变得更加容易。所以 $\mathrm{Sr}^{2+}$ 的
加入有助于推动晶粒的生长。 $\mathrm{NI}$ 等 ${ }^{[12]}$ 曾报道, 若添 加剂的量适中, 在 ZST 陶瓷烧结中形成的液相适量, 既有利于降低烧结温度, 又能充分排除气孔, 且不 至于留下过多的晶界相。LIU 等 ${ }^{[13]}$ 发现适量添加 $\mathrm{CuO}$ 和 $\mathrm{MoO}_{3}$ 能有效改善 $\mathrm{ZnO}-\mathrm{TiO}_{2}$ 微波介质陶瓷的 烧结性能, 这是因为 $\mathrm{CuO}$ 和 $\mathrm{MoO}_{3}$ 熔点较低, 在烧 结过程中形成适量的液相进而对陶瓷进行液相烧结 的结果。在本文中, 添加 $0.6 \mathrm{wt} \%$ 的 $\mathrm{SrO}$ 有助于促进 晶粒生长, 并且形成适量的液相, 而液相的形成降 低了试样的烧结温度, 提高了试样的致密度, 这表 明适量的液相对 ZST 陶瓷起到助熔和促进致密化烧 结的作用; 但随着 $\mathrm{SrO}$ 的添加量增加到 $0.8 \mathrm{wt} \%$ 、 $1.0 \mathrm{wt} \%$, 生成的液相过多会使晶粒生长速度过快, 大量的 $\mathrm{SrO}$ 存留在晶界中, 致使晶界移动速率过快, 使得许多气孔未来得及排出而残留在晶体内部, 从 而使试样的密度逐渐降低。而对比图 3(a、b) 可以看 出, 当 $\mathrm{MgO}$ 的添加量由 $0.2 \mathrm{wt} \%$ 增加到 $0.4 \mathrm{wt} \%$, 在 相同 $\mathrm{SrO}$ 的添加量下, 几乎所有试样的密度均有下 降。CHEN 等 ${ }^{[14]}$ 指出, $\mathrm{MgO}$ 添加量的增加有利于晶 粒的生长, 但是会导致 ZST 陶瓷的致密度下降。 PAMU 等 ${ }^{[15]}$ 研究 $\mathrm{MgO}$ 对 ZST 陶瓷密度的影响, 发 现在 $1350^{\circ} \mathrm{C}$ 烧结 $3 \mathrm{~h} 、 1400^{\circ} \mathrm{C}$ 烧结 2、3、4 h, 随着 $\mathrm{MgO}$ 添加量的增加, 陶瓷的密度逐渐减小, 这与本 研究的结论基本一致。

\section{4 高频介电性能}

图 4(a、b)为 $\mathrm{MgO}$ 的添加量为 $0.2 \mathrm{wt} \% 、 0.4 \mathrm{wt} \%$, 对应 $\mathrm{SrO}$ 添加量分别取 $0.2 \mathrm{wt} \% 、 0.4 \mathrm{wt} \% 、 0.6 \mathrm{wt} \%$ 、 $0.8 \mathrm{wt} \% 、 1.0 \mathrm{wt} \%$, 烧结温度范围为 $1280 \sim 1340^{\circ} \mathrm{C}$, 所 得试样的介电常数随着烧结温度的变化(测试频率 为 5 6 GHz)。从图 4 可以看到, 介电常数的变化规 律同密度的变化规律基本一致。当 $\mathrm{SrO}$ 的添加量为 $0.2 \mathrm{wt} \% 、 0.4 \mathrm{wt} \% 、 0.6 \mathrm{wt} \%$, 在 $1300{ }^{\circ} \mathrm{C}$ 烧结

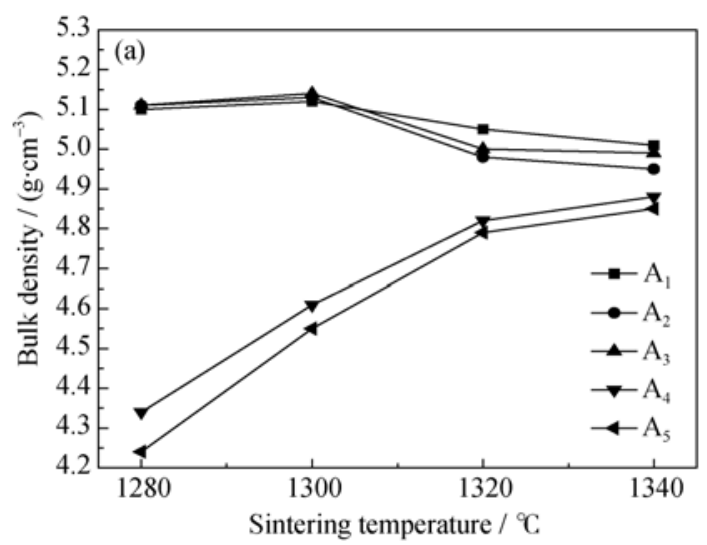

图 3 ZST 试样密度随烧结温度的变化

Fig. 3 Bulk densities of ZST specimens as a function of sintering temperature 

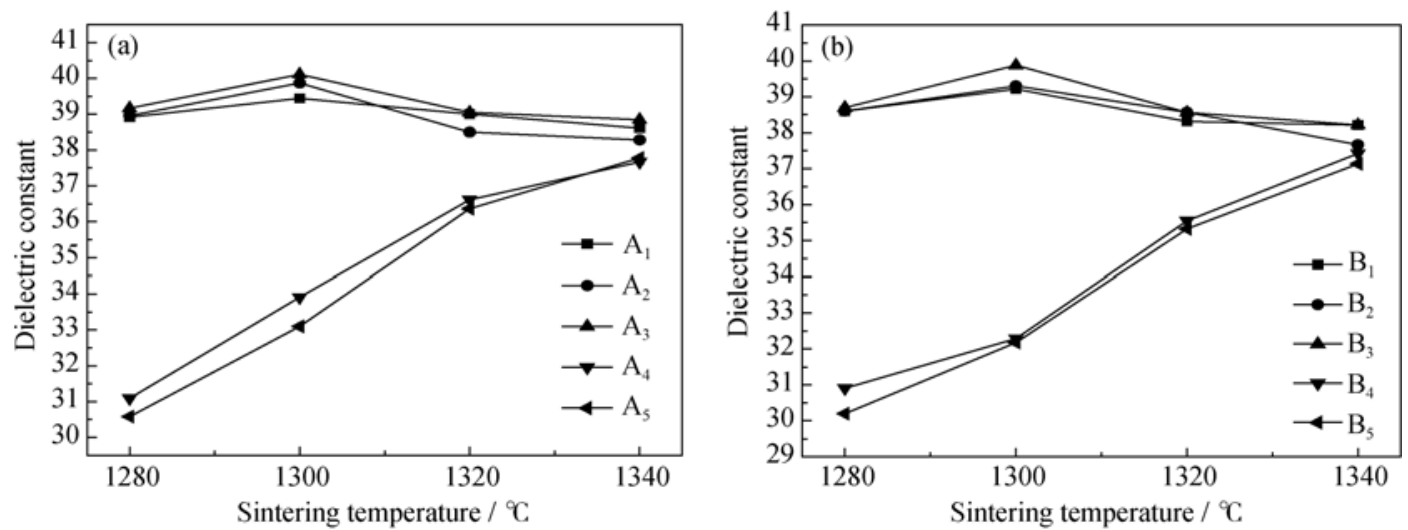

图 4 ZST 试样介电常数随烧结温度的变化

Fig. 4 Dielectric constants of the ZST specimens as a function of sintering temperature

时, $\mathrm{A}_{3}$ 试样与 $\mathrm{B}_{3}$ 试样的 $\varepsilon_{\mathrm{r}}$ 达到最大值, 分别为 40.11 和 39.88 。在烧结温度小于 $1300^{\circ} \mathrm{C}$ 时, 随着烧 结温度的升高, 试样的介电常数随之升高; 在烧结 温度大于 $1300^{\circ} \mathrm{C}$ 时, 随着烧结温度的继续升高, 试样的介电常数逐渐降低。当 $\mathrm{SrO}$ 的添加量为 $0.8 \mathrm{wt} \% 、 1.0 \mathrm{wt} \%$, 在 $1280^{\circ} \mathrm{C}$ 烧结时, $\mathrm{A}_{5}$ 试样的介电 常数仅为 $30.58, \mathrm{~B}_{5}$ 试样的介电常数仅为 30.2 ; 在 $1340^{\circ} \mathrm{C}$ 烧结时, $\mathrm{A}_{5} 、 \mathrm{~B}_{5}$ 的介电常数分别可以达到 37.78 和 37.13 。当烧结温度较低时, 介电常数较小, 随着烧结温度的升高, 试样的介电常数逐渐增大。 当烧结温度为 $1280^{\circ} \mathrm{C} 、 \mathrm{SrO}$ 添加量为 $0.8 \mathrm{wt} \%$ 、 $1.0 \mathrm{wt} \%$, 对于 ZST 陶瓷, 晶体结构中存在氧八面 体结构 ${ }^{[16-17]}$, 由于 $\mathrm{Sr}^{2+}$ 半径大于 $\mathrm{Zr}^{4+} 、 \mathrm{Sn}^{4+}$ 和 $\mathrm{Ti}^{4+}$, 这会导致 ZST 陶瓷晶胞体积的增大, 引起氧八面 体的扩张，在外场作用下使离子运动更加容易，介 电常数随之增大, 由表 2 也可以看出, $1280^{\circ} \mathrm{C}$ 烧结 的 $\mathrm{A}_{5}$ 试样, 其晶胞参数明显大于 $1300^{\circ} \mathrm{C}$ 烧结的 $\mathrm{A}_{1} 、 \mathrm{~A}_{3}$ 试样, 但实际上, 试样的介电常数却因为 $\mathrm{SrO}$ 添加量的增加而减小, 这是由于过量的 $\mathrm{SrO}$ 导 致烧结过程中物质迁移速度慢, 烧结过程结束后, 晶粒仍未完全生长, 主晶相合成不完全, 晶粒间气 孔较多, 陶瓷体未完全达到充分致密化, 这时气相 的所占比例较大, 因此 $\varepsilon_{\mathrm{r}}$ 较小; 而当烧结温度升高 至 $1340^{\circ} \mathrm{C}$, 晶粒比较完全的生长, 陶瓷体相对来 说已经较为致密, 因此 $\varepsilon_{\mathrm{r}}$ 有较大幅度的提高。对比 图 4(a、b) 可以看出, 当 $\mathrm{MgO}$ 添加量由 $0.2 \mathrm{wt} \%$ 增加 到 $0.4 \mathrm{wt} \%$, 在相同 $\mathrm{SrO}$ 的添加量下, 大部分试样的 介电常数有所下降。YU 等 ${ }^{[18]}$ 曾指出, 微波介质陶 瓷密度越高, 内部的离子参与极化的也越多, 在极 化率不变的前提下，必然使 $\varepsilon_{\mathrm{r}}$ 增大。由图 2(f)可以 看出, 当 $\mathrm{MgO}$ 的添加量由 $0.2 \mathrm{wt} \%$ 增加到 $0.4 \mathrm{wt} \%$,
试样内部形成的液相增多, 液相在冷却后常存在于晶 界处，这种晶界相往往具有较小的 $\varepsilon_{\mathrm{r}}$ 值; 烧结中结晶 速度过快会影响气孔的排除, 大量气孔陷于晶粒中, 会影响陶瓷的致密度，晶界相和气孔都会使 $\varepsilon_{\mathrm{r}}$ 下降。

图 5(a、b)为 $\mathrm{MgO}$ 的添加量为 $0.2 \mathrm{wt} \% 、 0.4 \mathrm{wt} \%$, 对应 $\mathrm{SrO}$ 添加量分别取 $0.2 \mathrm{wt} \% 、 0.4 \mathrm{wt} \% 、 0.6 \mathrm{wt} \%$ 、 $0.8 \mathrm{wt} \% 、 1.0 \mathrm{wt} \%$, 烧结温度范围为 $1280 \sim 1340^{\circ} \mathrm{C}$, 所 得试样的 $Q \times f$ 值随着烧结温度的变化(测试频率为 $5 \sim 6 \mathrm{GHz})$ 。在 $1300^{\circ} \mathrm{C}$ 烧结时, $\mathrm{A}_{3}$ 试样与 $\mathrm{B}_{3}$ 试样的 $Q \times f$ 值达到最大值, 分别为 51000 和 48100 。与密度, 介电常数变化规律基本类似，当 $\mathrm{SrO}$ 添加量为 $0.2 \mathrm{wt} \% 、 0.4 \mathrm{wt} \% 、 0.6 \mathrm{wt} \%$, 在 $1280^{\circ} \mathrm{C}$ 与 $1300^{\circ} \mathrm{C}$ 烧结 时, 试样 $Q \times f$ 值的变化不是很明显。当烧结温度高 于 $1300^{\circ} \mathrm{C}$ 时，试样的 $Q \times f$ 值有明显下降。当 $\mathrm{SrO}$ 添 加量为 $0.8 \mathrm{wt} \% 、 1.0 \mathrm{wt} \%$, 在烧结温度为 $1280^{\circ} \mathrm{C}$ 时, 试样的 $Q \times f$ 值非常低，仅为 12000 左右，而随着烧 结温度升高, 试样的 $Q \times f$ 值急剧增大。当烧结温度 较低、 $\mathrm{SrO}$ 添加量较大时, 由微波损耗理论可知, 晶格缺陷、杂质第二相、致密化程度和晶粒尺寸大 小等会影响陶瓷材料的非本征损耗 ${ }^{[19-20]}$ 。本研究中, ZST 试样在添加 $0.6 \mathrm{wt} \% \mathrm{SrO}$ 时, 晶粒生长均匀, 晶 格缺陷较少, 无杂相生成, 从而使试样的 $Q \times f$ 值较 高; 而当 $\mathrm{SrO}$ 的添加量为 $0.8 \mathrm{wt} \% 、 1.0 \mathrm{wt} \%$ 时，生成 的液相过多导致了晶粒尺寸的减小、密度的降低、 气孔率和缺陷的增加, 从而导致了试样 $Q \times f$ 值的减 小。对比图 5(a、b) 可以看出, 随着 $\mathrm{MgO}$ 添加量由 $0.2 \mathrm{wt} \%$ 增加到 $0.4 \mathrm{wt} \%$, 在相同 $\mathrm{SrO}$ 的添加量下, 试样 $Q \times f$ 值有所下降，但降低幅度不大。PAMU 等 ${ }^{[15]}$ 也指出, 在 $1400^{\circ} \mathrm{C}$ 保温 2、3、4 h, 随着 $\mathrm{MgO}$ 添 加量的增加, ZST 微波介质陶瓷的 $Q \times f$ 值明显降低, 这与本研究的结论基本类似。 

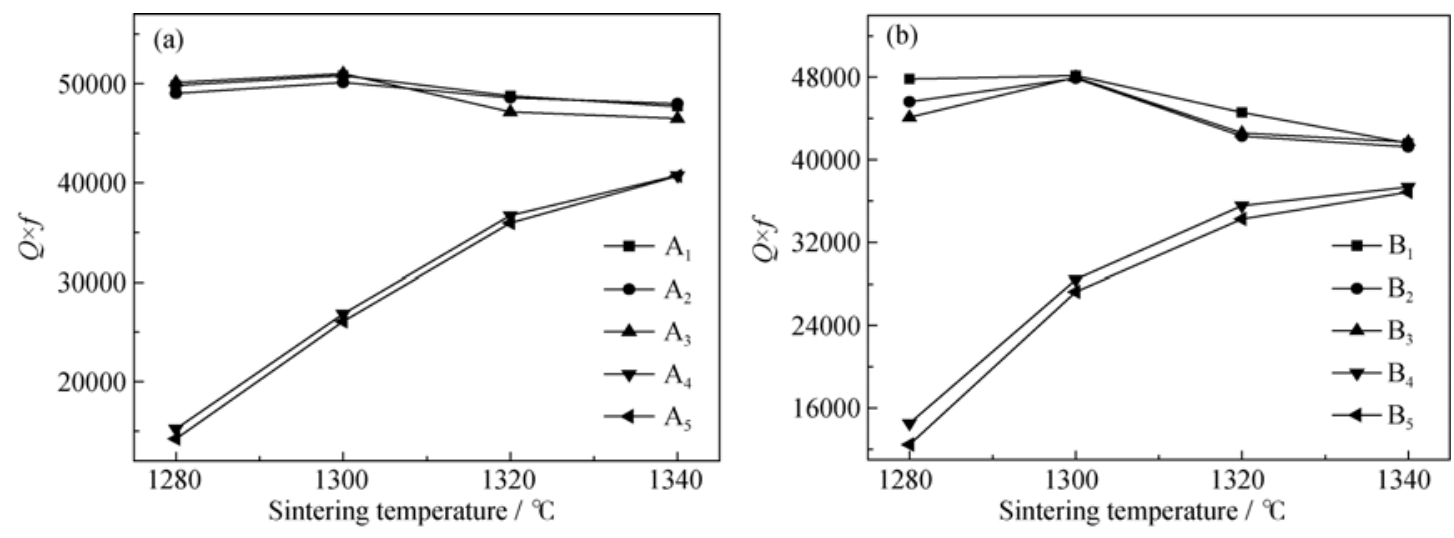

图 5 ZST 试样 $Q \times f$ 值随烧结温度的变化

Fig. $5 Q \times f$ values of ZST specimens as a function of sintering temperature

表 3 为不同 $\mathrm{MgO} / \mathrm{SrO}$ 添加量对试样频率温度系 数和抗弯强度的影响。试样的频率温度系数均为负 值, $1300^{\circ} \mathrm{C}$ 烧结的 $\mathrm{A}_{3}$ 试样数值最大, 其 $\tau_{\mathrm{f}}$ 为 $-2.85 \times$ $10^{-6} /{ }^{\circ} \mathrm{C} ; 1340^{\circ} \mathrm{C}$ 烧结的 $\mathrm{B}_{5}$ 试样的数值最小, 其 $\tau_{\mathrm{f}}$ 为 $-8.53 \times 10^{-6} /{ }^{\circ} \mathrm{C}$ 。随着添加剂添加量的变化, 试样的频 率温度系数有所变化, 但变化幅度不大, 并且数值 接近 0 。当 $\mathrm{MgO}$ 的添加量为 $0.2 \mathrm{wt} \% 、 0.4 \mathrm{wt} \%$, 随着 $\mathrm{SrO}$ 添加量的增加, 试样的抗弯强度是逐渐下降的。 $1300^{\circ} \mathrm{C}$ 烧结的 $\mathrm{A}_{1} 、 \mathrm{~B}_{1}$ 试样的抗弯强度最大, 分别为 $147.04 、 137.03 \mathrm{MPa}$; 当 $\mathrm{MgO}$ 的添加量由 $0.2 \mathrm{wt} \%$ 增 加到 $0.4 \mathrm{wt} \%$, 试样的抗弯强度有小幅度的降低。

\section{3 结论}

1) $\mathrm{MgO} / \mathrm{SrO} / \mathrm{La}_{2} \mathrm{O}_{3}$ 多元添加对 ZST 陶瓷的烧结 有一定的推动作用, 但 $\mathrm{SrO}$ 添加量的过大会导致陶 瓷密度下降和烧结温度的升高;

2) $\mathrm{SrO}$ 适量添加对 ZST 陶瓷的晶粒生长有较大 的推动作用, 但添加量过大会造成晶格缺陷和残留 气孔的增多, 从而导致材料的介电常数和 $Q \times f$ 值的 降低;

表 3 ZST 试样的频率温度系数和抗弯强度

Table 3 Frequency-temperature coefficient and flexure strength of ZST specimens

\begin{tabular}{cccc}
\hline Sample & $\begin{array}{c}\text { Sintering tem- } \\
\text { perature } /{ }^{\circ} \mathrm{C}\end{array}$ & $\begin{array}{c}\tau_{\mathrm{f}} / \\
\left(\times 10^{-6},{ }^{\circ} \mathrm{C}^{-1}\right)\end{array}$ & $\begin{array}{c}\text { Flex- } \\
\text { ure strength/ } \\
\mathrm{MPa}\end{array}$ \\
\hline $\mathrm{A}_{1}$ & 1300 & -5.14 & 147.04 \\
$\mathrm{~A}_{3}$ & 1300 & -2.85 & 143.61 \\
$\mathrm{~A}_{5}$ & 1340 & -6.14 & 131.34 \\
$\mathrm{~B}_{1}$ & 1300 & -4.46 & 137.03 \\
$\mathrm{~B}_{3}$ & 1300 & -4.26 & 135.56 \\
$\mathrm{~B}_{5}$ & 1340 & -8.53 & 125.45 \\
\hline
\end{tabular}

3) $\mathrm{MgO}$ 添加量的增大会导致 ZST 陶瓷密度、 介电常数、 $Q \times f$ 值和抗弯强度的下降;

4) $1300^{\circ} \mathrm{C}$ 保温 $5 \mathrm{~h}$, 复合添加 $0.2 \mathrm{wt} \% \mathrm{MgO}$ 、 $0.6 \mathrm{wt} \% \mathrm{SrO} 、 1.0 \mathrm{wt} \% \mathrm{La}_{2} \mathrm{O}_{3}$ 时, ZST 陶瓷的介电性能 最好, 其 $\rho=5.14 \mathrm{~g} / \mathrm{cm}^{3}, \varepsilon_{\mathrm{r}}=40.11, Q \times f=51000 \mathrm{GHz}$ $(f=5.61 \mathrm{GHz}), \tau_{\mathrm{f}}=-2.85 \times 10^{-6} /{ }^{\circ} \mathrm{C}$; 在 $1300^{\circ} \mathrm{C}$ 保温 $5 \mathrm{~h}$, 复合添加 $0.2 \mathrm{wt} \% \mathrm{MgO} 、 0.2 \mathrm{wt} \% \mathrm{SrO} 、 1.0 \mathrm{wt} \% \mathrm{La}_{2} \mathrm{O}_{3}$ 时, ZST 陶瓷的抗弯强度最好, 为 $147.04 \mathrm{MPa}$ 。

\section{参考文献:}

[1] CHEN TAO, WANG ZHE-FEI, HUANG BAO-YU, et al. Effects of annealing process on the properities and structure of $0.9 \mathrm{Al}_{2} \mathrm{O}_{3}$ $0.1 \mathrm{TiO}_{2}$ microwave dielectric ceramics. Journal of Synthetic Crystals, 2015, 44(2): 415-419.

[2] NARANG S B, BAHEL S. Low loss dielectric ceramics for microwave applications: a review. J. Ceram. Process. Res., 2010, 11(3): 316-321.

[3] WANG NING, ZHAO MEI-YU, YIN ZHI-WEN. Low-temperature firing in microwave dielectric ceramics. Journal of Inorganic Materials, 2002, 17(5): 916-924.

[4] WANG LIN-ZI, WANG LI-XI, WANG ZHE-FEI, et al. Effect of sintering aid $\mathrm{ZnO}-\mathrm{CeO}_{2}$ on dielectric properties of $\left(\mathrm{Zr}_{0.8} \mathrm{Sn}_{0.2}\right) \mathrm{TiO}_{4}$ ceramics. J. Mater. Sci. Mater. Electron., 2015, 26(11): 9026-9030.

[5] JACOB M V, PAMU D, RAJU K C J. Cryogenic microwave dielectric properties of sintered $\left(\mathrm{Zr}_{0.8} \mathrm{Sn}_{0.2}\right) \mathrm{TiO}_{4}$ doped with $\mathrm{CuO}$ and ZnO. J. Am. Ceram. Soc., 2007, 90(5): 1511-1514.

[6] QU JING-JING, WEI XING, JING BEN-QIN, et al. Microstructures and microwave dielectric properties of $(1-x)\left(\mathrm{Sr}_{0.2} \mathrm{Nd}_{0.208}\right.$ $\left.\mathrm{Ca}_{0.488}\right) \mathrm{TiO}_{3}-x \mathrm{Nd}\left(\mathrm{Ti}_{0.5} \mathrm{Mg}_{0.5}\right) \mathrm{O}_{3}$ ceramics with high quality factor. Journal of Inorganic Materials, 2015, 30(11): 1213-1217.

[7] 李标荣, 王花珍, 张绪礼. 无机电介质. 武昌: 华中理工大学出 版社, 1995: 154-166.

[8] NEDELCU L, IOACHIM A, TOACSAN M I, et al. Magnesium 
doped $(\mathrm{Zr}-0.8, \mathrm{Sn}-0.2) \mathrm{TiO}_{4}$ ceramics for microwave devices. $J$. Optoelectron. Adv. M., 2007, 9(6): 1727-1729.

[9] IOACHIM A, BANCIU M G, TOACSEN M I, et al. High-k Mg-doped ZST for microwave applications. Appl. Surf. Sci., 2006, 253(1): 335-338.

[10] ZHANG SHU-XIA, LI JIAN-BAO, ZHAI HUA-ZHANG, et al. Synthesis and characterization of $\mathrm{La}_{2} \mathrm{O}_{3} / \mathrm{BaO}$-doped $\left(\mathrm{Zr}_{0.8} \mathrm{Sn}_{0.2}\right) \mathrm{TiO}_{4}$ microwave ceramics. Ceram. Int., 2002, 28(4): 407-411.

[11] WANG GUO-QING, WU SHUN-HUA, ZHAO YU-SHUANG. Effects of dopants on the dielectric properties of $(\mathrm{Zr}, \mathrm{Sn}) \mathrm{TiO}_{4}$ system high-frequency ceramics. Bulletin of the Chinese Ceramic Society, 2003, 22(5): 7-12.

[12] NI JING, WU SONG-PING. Present study on adulteration properties of $(\mathrm{Zr}, \mathrm{Sn}) \mathrm{TiO}_{4}$ microwave dielectric ceramics. Journal of Ceramics, 2008, 29(2): 189-192.

[13] LIU ZHONG-CHI, ZHOU DONG-XIANG, GONG SHU-PING, et al. Sintering and phase transition of $0.25 \mathrm{CuO}-0.75 \mathrm{MoO}_{3}$ doped $\mathrm{ZnO}-\mathrm{TiO}_{2}$ microwave dielectric ceramics. Journal of Inorganic Materials, 2009, 24(4): 712-716.

[14] CHEN FEI, ZHOU HONG-QING, LIU MIN, et al. Effects of
$\mathrm{La}_{2} \mathrm{O}_{3}$ and $\mathrm{MgO}$ co-doping on properties of $\left(\mathrm{Zr}_{0.8} \mathrm{Sn}_{0.2}\right) \mathrm{TiO}_{4}$ ceramics. Journal of Functional Materials and Devices, 2010, 16(5): 479-482.

[15] PAMU D, RAO G L N, RAJU K C J. Effect of BaO, SrO and MgO addition on microwave dielectric properties of $\left(\mathrm{Zr}_{0.8} \mathrm{Sn}_{0.2}\right) \mathrm{TiO}_{4}$ ceramics. J. Alloys Compd., 2009, 475(1/2): 745-751.

[16] LIU LIN, FANG YOU-WEI, DENG XIN-FENG, et al. Crystal structures and microwave dielectric properties of $\left(\mathrm{Ba}_{1-x} \mathrm{Sr}_{x}\right)$ $\mathrm{La}_{4} \mathrm{Ti}_{4} \mathrm{O}_{15}(x=0.8-0.95)$ ceramics. Journal of Inorganic Materials, 2012, 27(3): 281-284.

[17] 王卓斌. $\left(\mathrm{Zr}_{0.8}, \mathrm{Sn}_{0.2}\right) \mathrm{TiO}_{4}$ 微波介质陶瓷材料工艺及应用. 四川: 电子科技大学硕士学位论文, 2012.

[18] YU XUAN, QIN WANG-YANG, JIA LU-FANG, et al. Effects of $\mathrm{ZnO}$ and $\mathrm{V}_{2} \mathrm{O}_{5}$ co-doping on properties of $\left(\mathrm{Zr}_{0.8} \mathrm{Sn}_{0.2}\right) \mathrm{TiO}_{4}$ ceramics. Electronics\&Packaging, 2015, 15(2): 40-43.

[19] HSUN C H, TSAI S H. Dielectric characteristics of Sr substitutionon $\mathrm{Ca}_{0.4} \mathrm{Sm}_{0.4} \mathrm{TiO}_{3}$ ceramics at microwave frequency. Ceram. Int., 2014, 40(7): 10111-10114.

[20] KUCHEIKO S, YEO D H, CHOI J W, et al. Microwave dielectric properties of $\mathrm{CaTiO}_{3}-\mathrm{Ca}\left(\mathrm{Al}_{1 / 2} \mathrm{Ta}_{1 / 2}\right) \mathrm{O}_{3}$ ceramics. J. Am. Ceram. Soc., 2002, 85(5): 2739-2740. 